\title{
On the possible ranks among matrices with a given pattern
}

by

Charles R. Johnson ${ }^{1}$

Department of Mathematics

College of William and Mary

Williamsburg, VA, 23187

\author{
YuLin Zhang ${ }^{2}$ \\ Centro de Matemática \\ Universidade do Minho \\ 4710 Braga, Portugal
}

\begin{abstract}
Given are tight upper and lower bounds for the minimum rank among all matrices with a prescribed zero-nonzero pattern. The upper bound is based upon solving for a matrix with a given null space and, with optimal choices, produces the correct minimum rank. It leads to simple, but often accurate, bounds based upon overt statistics of the pattern. The lower bound is also conceptually simple. Often, the lower and an upper bound coincide, but examples are given in which they do not.
\end{abstract}

AMS Classification: 15A03, 05C50.

Key Words: column rank stable, k-triangle, minimum rank, row gradual, superlinearly independent, totally nonzero null vector.

\section{Introduction}

If we know only the zero (nonzero) pattern of a real matrix, what ranks are possible? Given only the pattern, there is a large set of matrices, and the maximum rank (denoted by $M R$ ) possible has long been well understood [2]. It can be viewed, for example, as the minimum number of lines that cover all the nonzeros, or the greatest length of a transversal (equivalently the largest square submatrix that has a nonzero term in its determinant). If the minimum rank (denoted by $m r$ ) among our set of matrices is known, then all possible ranks are known, as every value of rank between $m r$ and $M R$ can occur. This is easily seen by changing a minimum rank matrix of the pattern to a maximum rank one, one entry at a time, and observing the

\footnotetext{
${ }^{1}$ Email address: crjohnso@MATH.WM.EDU

${ }^{2}$ Financial support provided by the Research Centre of Mathematics of the University of Minho through the FCT Pluriannual Funding Program. zhang@math.uminho.pt(corresponding author)
} 
ranks that occur. Since a rank 1 perturbation can change the rank by at most one, all intermediate ranks must appear along the way (perhaps with repeats and perhaps with decreases, as well as increases). Thus, understanding $m r$ (upon which we focus here) is equivalent to understanding all possible ranks. Unfortunately, $m r$ is quite a subtle problem, in general, as many have informally noted. We exhibit here natural lower and upper bounds on $m r$. The lower bound is quite simple (though we make important observation about it), while the upper bounds are more subtle. Often the lower and an upper bound coincide, rendering $m r$ clear. However, we note a 7-by-7 example (the smallest known), in which they differ. In this case $m r$ coincides with an upper bound. Other examples, indicating important limitations on our bounds are also given.

\section{Background and Notation}

By a pattern we mean an $m$-by- $n$ array $P$ of $0^{\prime} s$ and $*^{\prime} s$ in which the $*^{\prime} s$ are interpreted as nonzero real numbers whose values are, otherwise, free. Thus, we associate with $P$ the set of all $m$-by- $n$ real matrices $A=\left(a_{i j}\right)$ in which $a_{i j}=0$ if and only if the $i, j$ entry of $P$ is a 0 . Benignly, we use $P$ both for the formal pattern and to denote the collection of such matrices. Thus we might write $A \in P$ to indicate that $A$ has the indicated zero (nonzero) pattern. Much of what we say will also be valid over other fields, but historically, the qualitative interest in matricial properties associated with a pattern has centered upon the real field.

By $m r(P)(\operatorname{resp} ; M R(P))$ we mean $\min _{A \in P} \operatorname{rank}(A)\left(\operatorname{resp} ; \max _{A \in P} \operatorname{rank}(A)\right)$ the "minimum rank" (resp; "maximum rank") of $P$. As noted, all ranks between $m r(P)$ and $M R(P)$ occur in $P$, and $M R(P)$ is easily understood. Thus we concentrate upon $m r(P)$.

By a $k$-triangle we mean a $k$-by- $k$ pattern (or matrix) that is permutation equivalent to an upper triangular pattern with nonzero diagonal. In a $k$-triangle, we call the nonzeros that are on this diagonal the "key-nonzeros", and the zeros that end up below the diagonal, after permutation, the "key-zeros". The "maximum triangle size" is the maximum $k$-triangle that occurs as a subpattern of a given pattern. We will view "subpatterns" in the same formal way as is standard for submatrices. For example,

$$
\left[\begin{array}{lll}
* & 0 & * \\
* & 0 & * \\
0 & * & *
\end{array}\right]
$$

contains as a subpattern a 2-triangle in rows and columns 1 and 3, (and also in rows 1 and 3 and columns 2 and 3); the 3,1 entry is a "key-zero" and the 1,1 and 3,3 entries are "key-nonzeros". 
We close this section by noting, for reference, several simple properties of $m r$, of a pattern argument, that resemble those of rank. It should be noted that some basic properties of rank seem to have no natural generalization to $m r$ (which is one reason to note the ones that do). For example, if $A$ is an $m$-by- $n$ matrix of rank $k$, then there exist $k$ (particular) linearly independent rows of $A$ and a (particular) $k$-by- $k$ submatrix of full rank (a $k$-by- $k$ submatrix that determines the rank). For patterns, the analogous statements are not valid. For example, if $m r(P)=k$, there need be no particular $k$-rowed submatrix for which $m r$ is $k$. (This will be clear from examples to be given later.) Also, there is no analog of subadditivity for $m r$, with regard to two patterns of the same size.

We first note that $m r$ for a subpattern provides a lower bound for $m r$ of a pattern, just as with rank.

Proposition 2.1. If $Q$ is a subpattern of an $m$-by-n pattern $P$, then

$$
m r(Q) \leq m r(P) .
$$

Proof. For any matrix $A \in P$, we have $\operatorname{rank} B \leq \operatorname{rank} A$ if $B$ is a submatrix of $A$ in the positions of $Q$. So, $m r(Q) \leq \operatorname{rank} B \leq \operatorname{rank} A$. Now, choosing $A$ so as to achieve $m r(P)$ completes the proof.

Also, as with rank, removal of 0 or duplicate lines from a pattern does not change $m r$.

Proposition 2.2. If $P$ is an $m$-by-n pattern and $\hat{P}$ is the $(m-1)$-by-n $(m-b y-(n-1))$ pattern resulting from removal of an all zero row (column) or a row (column) that is identical to another row (column), then $m r(P)=m r(\hat{P})$.

Proof. The inequality $m r(P) \geq m r(\hat{P})$ follows from proposition 2.1. Now, if $\hat{A} \in \hat{P}$ achieves $m r(\hat{P})$, then return of the zero line, or the duplicate line with actual numerical values duplicated, to $\hat{A}$ to give $A \in P$, shows that $m r(P) \leq m r(\hat{P})$ and completes the proof.

In obtaining upper bounds for $m r$, the following can be helpful.

Proposition 2.3. Suppose that, partitioned by rows (columns)

$$
P=\left[\begin{array}{lll}
\ldots & P_{1} & \ldots \\
\ldots & P_{2} & \ldots
\end{array}\right], \text { or } P=\left[\begin{array}{cc}
\vdots & \vdots \\
P_{1} & P_{2} \\
\vdots & \vdots
\end{array}\right]
$$

then $m r(P) \leq m r\left(P_{1}\right)+m r\left(P_{2}\right)$. 
Proof. Apply the corresponding inequality for rank to $m r$-achieving matrices $A_{1} \in P_{1}$ and $A_{2} \in P_{2}$ and note that

$$
\left[\begin{array}{ccc}
\ldots & A_{1} & \cdots \\
\ldots & A_{2} & \cdots
\end{array}\right] \in P
$$

With the partitioning in proposition 2.3, it follows from proposition 2.1 and proposition 2.3 that

$$
\max \left\{m r\left(P_{1}\right), m r\left(P_{2}\right)\right\} \leq m r(P) \leq m r\left(P_{1}\right)+m r\left(P_{2}\right)
$$

and, in particular, that

$$
m r(P) \leq m r\left(P_{1}\right)+k,
$$

in which $k$ is the number of rows (columns) of $P_{2}$.

Equality can occur in proposition 2.3, for example, in case there is a line with just one nonzero.

Proposition 2.4. Suppose that,

$$
P=\left[\begin{array}{cc}
* & \ldots \ldots \\
0 & \\
\vdots & P_{1} \\
0 &
\end{array}\right]
$$

Then $m r(P)=1+m r\left(P_{1}\right)$.

Thus, if there is a line of $P$ with only one nonzero, then $m r(P)=1+m r(\tilde{P})$, in which $\tilde{P}$ is the subpattern complementary to the lone nonzero. Of course, equality also occurs for "direct sums".

Proposition 2.5. If $P$ is an $m$-by-n pattern, while $P_{1}$ is $m_{1}$-by- $n_{1}$ and $P_{2}$ is $m_{2}$-by$n_{2}$, with $m=m_{1}+m_{2}$ and $n=n_{1}+n_{2}$,

$$
P=\left[\begin{array}{cc}
P_{1} & 0 \\
0 & P_{2}
\end{array}\right],
$$

then $m r(P)=m r\left(P_{1}\right)+m r\left(P_{2}\right)$.

It is also important that $P_{1}$ and $P_{2}$ are non-overlapping and that any row of $P$ among those of $P_{1}$ is (combinatorially) orthogonal to any for $P_{2}$. 
It is not generally a simple matter to replace the problem of $m r(P)$ by that of minimum rank of a lower dimensional pattern through any kind of elimination process (with the exception of proposition 2.4): However, replacement by several minimum rank problems is, in principle, possible. As this is not often fruitful, we only give an indicative example.

Suppose that

$$
P=\left[\begin{array}{ccccc}
* & * & 0 & * & * \\
* & * & * & 0 & * \\
0 & & & & \\
\vdots & & P_{1} & & \\
0 & & & &
\end{array}\right]
$$

For any given $A \in P$, we might use the (nonzero) 1,1 entry to eliminate the 2,1 entry. However, the zero/nonzero status of 2,2 and 2,5 entries are then ambiguous, though if nonzero, they are arbitrary. Thus,

$$
m r(P)=1+\min \left\{m r\left(P_{2}\right), m r\left(P_{2}^{\prime}\right), m r\left(P_{5}\right), m r\left(P_{5}^{\prime}\right)\right\},
$$

in which

$$
\begin{array}{lll}
P_{2}=\left[\begin{array}{cccc}
* & * & * & 0 \\
& P_{1} & &
\end{array}\right], & P_{2}^{\prime}=\left[\begin{array}{cccc}
0 & * & * & 0 \\
& P_{1} & &
\end{array}\right], \\
P_{5}=\left[\begin{array}{cccc}
* & * & * & * \\
& P_{1} & &
\end{array}\right], & P_{5}^{\prime}=\left[\begin{array}{cccc}
0 & * & * & * \\
& P_{1} & &
\end{array}\right] .
\end{array}
$$

In more complicated situations, care must be taken to avoid new patterns with correlated entries if the row used to eliminate has nonzeros where rows, in which elimination occurs, have zeros.

\section{Upper Bounds for Minimum Rank}

In order that an $m$-by- $n$ matrix $A$ have rank $\leq n-k$, the orthogonal complement of the rows of $A$ must be at least $k$-dimensional, and, thus must have a basis of at least $k$-vectors. Here, for a given pattern $P$, we consider the inverse-problem of our problem. We first prescribe a collection of linearly independent vectors and then attempt to design a matrix of the pattern whose null space includes these vectors. If successful, this immediately gives an upper bound for $m r(P)$. Gradual refinement of this idea leads to increasingly subtle bounds.

In order that (linearly independent) vectors $y_{1}, y_{2}, \ldots, y_{k}$ be in the null space of $A$, a certain homogeneous linear system, whose coefficients arise from entries of the 
$y^{\prime} s$, must be satisfied by the nonzero entries of $A$. Fixing the $y^{\prime} s$ we regard the entries of $A$ as variables. If we wish that $A \in P$, there is a variable for each nonzero of $P$, and we are interested in the existence of a "totally nonzero" (every variable nonzero) solution. Of course, a good choice of the $y^{\prime} s$ is important, but what is the form of this coefficient matrix?

Let $x_{P}$ be the vector of variables associated with the nonzeros of pattern $P$, numbered left-to-right within rows, beginning with the first row. Call the coefficient matrix of the homogeneous linear system that expresses that $y_{1}, y_{2}, \ldots, y_{k}$ are in the null space of $A \in P, B_{k}=B_{k}\left(y_{1}, y_{2}, \ldots, y_{k}\right)$. For example, if

$$
\begin{aligned}
& P=\left[\begin{array}{lll}
* & * & 0 \\
* & * & * \\
* & 0 & *
\end{array}\right]=\left[\begin{array}{ccc}
x_{11} & x_{12} & 0 \\
x_{21} & x_{22} & x_{23} \\
x_{31} & 0 & x_{33}
\end{array}\right] \\
& \text { and } y_{1}=\left[\begin{array}{l}
y_{11} \\
y_{12} \\
y_{13}
\end{array}\right], y_{2}=\left[\begin{array}{l}
y_{21} \\
y_{22} \\
y_{23}
\end{array}\right] \text {, for } k=2 \text {, we have } \\
& B_{2}=\left[\begin{array}{cccccccc}
y_{11} & y_{12} & 0 & 0 & 0 & 0 & 0 \\
0 & 0 & y_{11} & y_{12} & y_{13} & 0 & 0 \\
0 & 0 & 0 & 0 & 0 & y_{11} & y_{13} \\
y_{21} & y_{22} & 0 & 0 & 0 & 0 & 0 \\
0 & 0 & y_{21} & y_{22} & y_{23} & 0 & 0 \\
0 & 0 & 0 & 0 & 0 & y_{21} & y_{23}
\end{array}\right] .
\end{aligned}
$$

Our convention is that the initial $n$ rows of $B_{k}$ correspond to $y_{1}$, etc, Thus $B_{k}$ has $k n$ rows if $P$ is $m$-by-n and as many columns as the number of nonzeros in $P$. If $B_{k} x_{P}=0$ has a totally nonzero solution, then there is a matrix in $P$ whose null space includes $y_{1}, y_{2}, \ldots, y_{k}$; if $y_{1}, y_{2}, \ldots, y_{k}$ are linearly independent, then the rank of this matrix is at most $n-k$, and $m r(P) \leq n-k$.

Matrices with totally nonzero null vectors have been studied (for a different purpose) in [1]. One of the equivalent conditions found there is that $B$ is such a matrix if and only if the deletion of no single column of $B$ decreases its rank. Here we call such a matrix column rank stable. Of course row rank stable, then, also has a natural meaning and characterizes the existence of a left, totally nonzero null vector. The two concepts are not equivalent.

We summarize the above discussion in the following theorem. The first four statements are obviously equivalent, given the discussion and notation, and the equivalence of (4) and (5) follows from lemma 7(d) of [1]. See [1] for other equivalent statements.

Theorem 3.1. For an $m$-by-n pattern $P$, the following statements are equivalent: 
(1) $m r(P) \leq n-k$;

(2) there is an $A \in P$ such that $\operatorname{rank} A \leq n-k$;

(3) there exist $A \in P$ and $k$ linearly independent vectors $y_{1}, y_{2}, \ldots, y_{k}$ such that $y_{i}$ lies in the null space of $A, i=1, \ldots, k$;

(4) there exist linearly independent vectors $y_{1}, y_{2}, \ldots, y_{k}$ such that $B_{k}\left(y_{1}, y_{2}, \ldots, y_{k}\right) x_{p}=$ 0 has a totally nonzero solution; and

(5) there exist $y_{1}, y_{2}, \ldots, y_{k}$, linearly independent, such that $B_{k}\left(y_{1}, y_{2}, \ldots, y_{k}\right)$ is column rank stable.

It should be noted that item (5) can give the precise minimum rank if a correct collection of $y^{\prime} s$ is chosen; the theorem may be viewed as a duality between minimum rank and maximum nullity. As we shall see this provides a powerful tool in the analysis of $m r$. It is very important that the form of $B_{k}$ is independent of the particular $y^{\prime} s$ and that, if there are sufficiently many $y^{\prime} s$, the column rank stable property forces the collection of subvectors in certain positions to be linearly dependent. We may use Theorem 3.1 to obtain, first a very simple upper bound for $m r(P)$. Let $r_{1}, \ldots, r_{m}$ be the numbers of nonzeros in the rows of the $m$-by-n pattern $P$, listed in increasing order: $r_{1} \leq r_{2} \leq \ldots \leq r_{m}$ (not according to row number). Similarly $c_{1} \leq c_{2} \leq \ldots \leq c_{n}$ are the numbers of nonzeros in the columns. Of course, $P$ is permutation equivalent to a pattern in which the subscripts on the $r^{\prime} s$ and $c^{\prime} s$ also refer to the row (column) number. If $P$ has a lot of nonzeros, it is (roughly) easier to find a low rank matrix in $P$, and it is this intuition that we wish to quantify.

To this end, we say that a collection of $k$ vectors $y_{1}, y_{2}, \ldots, y_{k} \in R^{n}$ is super-linearly independent (SLI) if every collection of $k$ vectors, formed by taking subvectors of them in any $k$ specific positions, is linearly independent. Of course, $k \leq n$ and it follows that subvectors formed from more than $k$ positions are linearly independent. Since totally positive matrices of any size (e.g. $n$-by- $k$ ) are known to exist (and in any event super-linear independence is generic), we have the following fact,

Lemma 3.2. For each $k \leq n$, there exist $k$ SLI vectors in $R^{n}$.

Suppose now that for a given pattern $P, r_{1}=k+1 \geq 2$, and that $y_{1}, y_{2}, \ldots, y_{k}$ are SLI. Then, $B_{k}\left(y_{1}, y_{2}, \ldots, y_{k}\right)$ is full row rank $\left(\operatorname{rank} B_{k}=k n\right)$ and is column rank stable. This is easily seen because the contiguous columns of $B$ associated with a given row of $P$ are all $0^{\prime} s$, except for a $k$-by- $r_{i}$ submatrix that is full row rank; all other entries of $B_{k}$ in the rows of this block are 0 . Since $r_{i} \geq r_{1}>k$ and because of the SLI property, any single-column-deleted submatrix also has rank $k$. Thus the rows of $B_{k}$ and of any single-column-deleted submatrix are linearly independent. Since $B_{k}$ is column rank stable and $y_{1}, y_{2}, \ldots, y_{k}$ are linearly independent, using theorem 3.1 parts 1 and 5 , we may conclude 
Corollary 3.3. If $P$ is an $m$-by-n pattern, then

$$
m r(P) \leq\left\{\begin{array}{c}
n+1-r_{1} \\
m+1-c_{1}
\end{array}\right.
$$

in which $r_{1}$ (resp, $c_{1}$ ) is the smallest number of nonzeros in a row (resp, column) of $P$.

Of course, the bound depending upon $c_{1}$ is proven, for example, by replacing $P$ by its transpose and applying the bound based upon $r_{1}$. It is possible that one gives a smaller lower bound than the other, as in, for example, the pattern:

$$
\left[\begin{array}{lll}
* & * & 0 \\
0 & * & * \\
0 & * & *
\end{array}\right]
$$

With some simple observations, corollary 3.3 may be noticeably refined with some simple observations. The point of corollary 3.3 is that if all the rows of a pattern have many nonzeros, then at the minimum, there is noticeable column rank deficiency. By partitioning $P$ by groups of rows, additional insight might be gained. Let

$$
\left[\begin{array}{ccc}
\ldots & P_{1} & \ldots \\
\ldots & \ldots & \ldots \\
\ldots & P_{p} & \ldots
\end{array}\right]
$$

in which $P_{i}$ is $m_{i}$-by- $n, i=1, \ldots, p$ and $\sum m_{i}=m$. Of course, it is clear, using 2.3 that

Lemma 3.4. We have

$$
m r(P) \leq \sum_{i=1}^{p} m r\left(P_{i}\right)
$$

The potential advantage of lemma 3.4 is that, not only may either bound in corollary 3.3 be used on each strip $P_{1}, \ldots, P_{p}$, but within each strip duplicate or zero columns may be deleted ( as may duplicate or zero rows/columns in the entire pattern). For example, if

$$
P=\left[\begin{array}{llll}
* & 0 & 0 & 0 \\
0 & * & * & 0 \\
0 & 0 & * & * \\
0 & * & 0 & *
\end{array}\right],
$$

then corollary 3.3 gives only the bound $m r(P) \leq 4$, but with lemma $3.4, m r(P) \leq 3$ is seen by letting $P_{1}$ be the first row and $P_{2}$ be the rows $2,3,4$. 
Sample general applications follow.

Given $r_{1} \leq \ldots \leq r_{m}$ and $c_{1} \leq \ldots \leq c_{n}$ for the $m$-by- $n$ pattern $P$, let

$$
d_{i}^{r}=r_{i}-i \quad \text { and } \quad d_{j}^{c}=c_{j}-j, i=1, \ldots m, j=1, \ldots, n .
$$

Then let

$$
d^{r}=\max _{1 \leq i \leq m} d_{i}^{r} \quad \text { and } \quad d^{c}=\max _{1 \leq j \leq n} d_{j}^{c} .
$$

Consider, for example, the rows. Suppose that they are numbered so that $r_{i}$ is the number of nonzeros in row $i$ and that $d_{r}=d_{i}^{r}$ for a particular $i$. Then let $P_{1}$ be the first $i-1$ rows and $P_{2}$ the remaining rows

$$
P=\left[\begin{array}{lll}
\ldots & P_{1} & \cdots \\
\ldots & P_{2} & \cdots
\end{array}\right]
$$

Of course $m r\left(P_{1}\right) \leq i-1$, and, by corollary $3.3, \operatorname{mr}\left(P_{2}\right) \leq n-r_{i}+1$, so that by lemma 3.4, $\operatorname{mr}(P) \leq i-1+n-r_{i}+1=n-\left(r_{i}-i\right)=n-d_{i}^{r}=n-d^{r}$. As, again, the same argument applies to the transpose of $P$, we have

Theorem 3.5. For an $m$-by-n pattern $P$,

$$
m r(P) \leq\left\{\begin{array}{c}
n-d^{r} \\
m-d^{c}
\end{array}\right.
$$

This, of course, is a formal improvement upon corollary 3.3. Suppose now that $P$ again is partitioned by block rows

$$
\left[\begin{array}{ccc}
\ldots & P_{1} & \ldots \\
\ldots & \ldots & \ldots \\
\ldots & P_{p} & \ldots
\end{array}\right]
$$

and let $\tilde{P}_{i}$ be $P_{i}$ with all zero lines and duplicate rows/columns removed. If $\tilde{P}_{i}$ is $\tilde{m}_{i}$-by- $\tilde{n}_{i}$, we have

Corollary 3.6. Let $P$ be a pattern partitioned as above. Then

$$
\operatorname{mr}(P) \leq \sum_{i=1}^{p} \min \left\{\tilde{n}_{i}-d^{r}\left(\tilde{P}_{i}\right), \tilde{m}_{i}-d^{c}\left(\tilde{P}_{i}\right)\right\} .
$$


Example. Let

$$
P=\left[\begin{array}{llllll}
* & * & * & 0 & 0 & 0 \\
* & * & * & 0 & 0 & 0 \\
* & * & * & 0 & 0 & 0 \\
0 & * & 0 & * & * & 0 \\
0 & * & * & 0 & * & * \\
0 & 0 & * & * & 0 & *
\end{array}\right]
$$

The best that may be said from corollary 3.3 and theorem 3.5 is that $m r(P) \leq 4$. However, partitioning with $P_{1}$ the first three and $P_{2}$ the last three rows gives $m r(P) \leq$ $m r\left(P_{1}\right)+m r\left(P_{2}\right) \leq 1+2=3$, which may be seen to be the minimum rank using ideas of the next section.

\section{A lower bound for minimum rank}

An important subtlety of the study of minimum rank is that $m r$ (of a pattern) obeys some of the same properties as rank and not others. For example, if a matrix has rank $k$, there are $k$ particular rows that are necessarily independent. If $m r(P)=k$, not only is there is no reason there should exist a $k$-rowed submatix $G$ of $P$ such that $\operatorname{mr}(G)=k$, but it can occur that there not be one (see section 5). However, if $P$ does have a subpattern $Q$ for which $m r(Q)=k$, then $m r(P) \geq k$. Because every $A \in P$ has only one nonzero term in its determinant when $P$ is a $k$-triangle, we have

Lemma 4.1. If $P$ is a k-triangle, $m r(P)=k$.

Interestingly, the converse is also valid.

Theorem 4.2. Let $P$ be an $m$-by-n pattern, $m \leq n$. Then $m r(P)=m$ if and only if $P$ contains an $m$-triangle.

Proof: we need only verify the forward implication. Assume $m r(P)=m$. Assuming without loss of generality, that $P$ has no 0 columns, it follows that $c_{1}(P)=1$. If $c_{1}(P)$ were $\geq 2$, then by corollary $3.3, \operatorname{mr}(P)$ would be less than $m$. Now suppose, via permutation equivalence, that the 1,1 entry of $P$ is a $*$ and that it is the only $*$ in the first column. Then, $\operatorname{mr}(P)=1+m r\left(P_{1}\right)$ in which $P_{1}$ is the result of deletion of the first row and column from $P$. Thus, $P_{1}$ is $(m-1)$-by- $(n-1)$ and $m r\left(P_{1}\right)=m-1$. By induction (initial cases are easily checked), $P_{1}$ contains an $(m-1)$-triangle. Return of the first column of $P$, and the appropriate entries in the first row, extends this $(m-1)$-triangle to an $m$-triangle lying in $P$.

Since $m r(Q) \leq m r(P)$ whenever $Q$ is a subpattern of $P$, we have 
Theorem 4.3. If $P$ is an $m$-by-n pattern that contains a $k$-triangle, $k \leq m, n$, then

$$
k \leq m r(P) .
$$

For patterns with small $m, n$ (e.g $\leq 5)$, the converse may be verified exhaustively. As we will see in the next two sections, the converse is not valid in general, even for square patterns and even for $k=n-1$. The examples are, likely, minimal.

\section{A Constraining Example}

For many patterns, the lower bound of section 4 and an upper bound from section 3 coincide, so that minimum rank is determined. In fact, the minimum rank is often the maximum triangle size. However, this is not always so, and our first purpose in this section is to exhibit an example (7-by-7, likely the smallest). Ron Holzmann suggested trying this pattern, and we thank him for the suggestion.

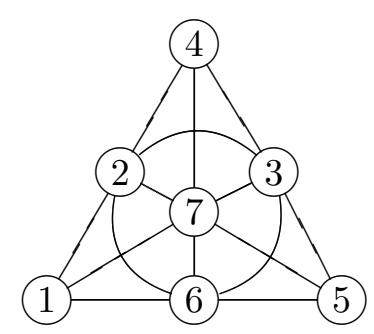

The projective plane of order 2 involves the incidence relationship between 7 points and 7 "lines". As every point lies on 3 lines and every line is determined by just three points, the incidence matrix is a $0-1,7$-by- 7 matrix with row and column sums 3. Our pattern is the complement of this incidence matrix, ie, the $0^{\prime} s$ of the incidence matrix become $*^{\prime} s$ (nonzeros) and the $1^{\prime} s$ become zeros. Specifically, our pattern $P$ may be presented (up to permutation of rows and columns) as

$$
P=\left[\begin{array}{lllllll}
* & * & * & 0 & * & 0 & 0 \\
0 & * & 0 & * & * & * & 0 \\
0 & 0 & * & 0 & * & * & * \\
* & 0 & * & * & 0 & * & 0 \\
0 & * & * & * & 0 & 0 & * \\
* & 0 & 0 & * & * & 0 & * \\
* & * & 0 & 0 & 0 & * & *
\end{array}\right]
$$

with 4 nonzeros in every row and column. 
An inspection of $P$ shows that it includes (many) 3-triangles, but no 4-triangles. thus, $3 \leq m r(P)$. On the other hand, since $r_{1}=c_{1}=4$ (4 nonzeros in every line), $m r(P) \leq 7-4+1=4$. No partitioning of $P$ yields a better estimate; in fact, any partition of the rows (columns) into two groups yields a higher sum of minimum ranks estimate. This is not surprising as all collections of 3 rows contain a 3 -triangle. In any event, we have

$$
3 \leq m r(P) \leq 4 .
$$

Which is it: 3 or 4 ? Our purpose is to show $\operatorname{mr}(P)=4$, by ruling out the possibility of 3. Suppose that $A \in P$ and $\operatorname{rank} A=3$. Because the first 3 rows of $P$ contain (two, non-overlapping) 3 -triangles, the first 3 rows of $A$ must be linearly independent, so that each of rows 4 through 7 is a linear combination of rows 1 through 3 . We write this down carefully, keeping in mind the zeros and nonzeros, to see what it means.

Since rank is unchanged by diagonal equivalence, we may suppose that the first 3 rows of $A$ appear as

$$
\left[\begin{array}{ccccccc}
1 & a_{12} & a_{13} & 0 & 1 & 0 & 0 \\
0 & 1 & 0 & 1 & a_{25} & 1 & 0 \\
0 & 0 & 1 & 0 & a_{35} & a_{36} & 1
\end{array}\right]
$$

in which $a_{12}, a_{13}, a_{25}, a_{35}$ and $a_{36}$ are nonzeros, as we may normalize some entry in each column (a bit more normalization is possible, but not convenient).

Since row $k$ of $A, k=4, \ldots, 7$, is a unique linear combination of the first 3 rows, we let $x_{k}, y_{k}$ and $z_{k}$, respectively, be the coefficients of rows 1,2 and 3 in the linear combination that gives row $k, k=4, \ldots, 7$. An inspection of these linear combinations and the zero/nonzero pattern of $P$ yields that $z_{4}, x_{5}$, and $y_{7}$ are all 0 , while the remaining coefficients must be nonzeros. For example $z_{4}$ is the last entry of row 4 (because the last entries of rows 1 and 2 are 0 ), which is 0 . On the other hand $y_{5}$ is the second entry of row 5 (because $x_{5}$ is 0 ), which is a nonzero, so that $y_{5}$ must be nonzero.

Now, to explain the remaining zeros in row 4 through 7 , there are homogeneous linear relations involving the $x, y$ and $z$ with a given subscript. These are

and

$$
\begin{array}{ccll}
a_{12} x_{4}+y_{4} & =0 & & x_{4}+a_{25} y_{4}=0 \\
a_{25} y_{5}+a_{35} z_{5} & =0 & & y_{5}+a_{36} z_{5}=0 \\
a_{13} x_{7}+z_{7}= & = & x_{7}+a_{35} z_{5}=0 \\
a_{12} x_{6}+y_{6}=0 \quad a_{13} x_{6}+z_{6}=0 & y_{6}+a_{36} z_{6}=0 .
\end{array}
$$

Written as linear systems, these become

$$
\left[\begin{array}{cc}
a_{12} & 1 \\
1 & a_{25}
\end{array}\right]\left[\begin{array}{l}
x_{4} \\
y_{4}
\end{array}\right]=\left[\begin{array}{l}
0 \\
0
\end{array}\right], \quad\left[\begin{array}{cc}
a_{25} & a_{35} \\
1 & a_{36}
\end{array}\right]\left[\begin{array}{l}
y_{5} \\
z_{5}
\end{array}\right]=\left[\begin{array}{l}
0 \\
0
\end{array}\right],
$$




$$
\left[\begin{array}{ccc}
a_{12} & 1 & 0 \\
a_{13} & 0 & 1 \\
0 & 1 & a_{36}
\end{array}\right]\left[\begin{array}{l}
x_{6} \\
y_{6} \\
z_{6}
\end{array}\right]=\left[\begin{array}{l}
0 \\
0 \\
0
\end{array}\right], \quad\left[\begin{array}{cc}
a_{13} & 1 \\
1 & a_{35}
\end{array}\right]\left[\begin{array}{l}
x_{7} \\
z_{7}
\end{array}\right]=\left[\begin{array}{l}
0 \\
0
\end{array}\right]
$$

If any of the coefficient matrices were nonsingular, the indicated $x^{\prime} s, y^{\prime} s$ and $z^{\prime} s$ could not be totally nonzero. So, we conclude that the determinant of each matrix must be 0 . This means:

$$
\begin{aligned}
& a_{12} a_{25}=1 \\
& a_{25} a_{36}=a_{35} \\
& -a_{13} a_{36}=a_{12} \text {. } \\
& a_{13} a_{35}=1
\end{aligned}
$$

Substituting the equations for $a_{12}$ and $a_{35}$ into the other two equations, gives

$$
a_{13} a_{36} a_{25}=-1, \quad \text { and } \quad a_{13} a_{25} a_{36}=1 .
$$

Since $1 \neq-1$, this contradiction shows that $m r(P)=3$ is not the case. Thus, $m r(P)=4$. We note that this contradiction occurs for any field other than $G F_{2}$; indeed, for $G F_{2}$ there is no contradiction and the minimum rank would be 3 . The (unique) 0,1 matrix of the pattern has rank 3 .

It is interesting to view this example in light of theorem 4.2. If rank 3 were possible, $B_{4}\left(y_{1}, y_{2}, y_{3}, y_{4}\right)$ would be 28 -by- 28 . If the $y^{\prime} s$ were SLI, then $B_{4}$ would be full rank and could not be column rank stable. So the $y^{\prime} s$ could not be SLI. In fact, because of the block structure of $B_{4}$ (permutable to a direct sum of 74 -by-4's, each of which corresponds to subvectors, of 4 components, of the $y^{\prime} s$ ), each collection of 4-component subvectors of $y_{1}, y_{2}, y_{3}, y_{4}$ that corresponds to the nonzeros in a row of $P$ would have to be linearly dependent. When this occurs, it can not both happen that the $y^{\prime} s$ are linearly independent and $B_{4}$ is column rank stable ( because of theorem 3.5 and because we have ruled out rank 3 for this pattern). We do not know if linear dependence for these 7 collections without linear dependence of the $4 y^{\prime} s$ is possible, but it appears not over, say, the real field. Apparently it is possible over $G F_{2}$.

\section{Minimal Rank $n-1$}

It is an interesting question just to characterize those patterns for which $m r=$ $n-1$. Of course, for such $m$-by- $n$, we may suppose that $m \geq n$ because of theorem 4.2. But, even for $m=n$ (and $k=n-1$ ), the converse of theorem 4.3 is not valid, as we shall see here. 
It will be important to recognize when there is an $(n-1)$-triangle present. If there is a row with just one nonzero, this problem (and that of $m r$ by proposition 2.4) reduces to that in a smaller pattern. If $m=n$, there cannot be an $(n-1)$-triangle if there are at least 3 nonzeros in each row. Thus, we assume, here, that $r_{1}$, the minimum number of nonzeros in a row is exactly 2 . To look for $(n-1)$-triangles, we introduce a notion of "row gradual" for a pattern $P$. This notion could be parameterized by the size of the triangle (and be initiated with a row with more than 2 nonzeros), but we focus upon $n-1$ and do not pursue the fairly obvious generalization here.

Let $P$ be an $m$-by-n pattern, $m \geq n$, and let $R_{i}$ denote the set of column indices of the nonzero positions in row $i$.

The pattern is said to be row gradual if (a) there is at least one row $\left(i_{1}\right)$ with exactly 2 nonzeros and (b) there is a sequence of rows $i_{1}, i_{2}, \ldots, i_{n-1}$ such that, for $S_{1}=R_{i_{1}}$ and $S_{j+1}=S_{j} \cup R_{i_{j+1}}, j=1, \ldots, n-2$, we have $\left|S_{j}\right|=j+1$. A notion of "column gradual" is analogous.

We now have three related concepts: $m r \geq n-1$, the existence of an $(n-1)$ triangle and the notion of row (column) gradual. The question is: what are the relationships among them and what role does the value of $m$ relative to $n$ play? Several observations are clear.

Proposition 6.1. If the pattern $P$ is row gradual, then $P$ contains an $(n-1)$-triangle

We note that the key nonzeros of this triangle are indicated by the indices added by each successive $S_{j}$, along with one of the indices in $R_{i_{1}}$. For this $m \geq n-1$ is all that is necessary. Of course if $P$ is row gradual, any row and/or column permutation of $P$ is as well. From proposition 6.1 and theorem 4.3, we then have

Corollary 6.2. If the pattern $P$ is row gradual, then $m r(P) \geq n-1$.

The above corollary may be viewed as a converse to theorem 3.5.

Conversely to proposition 6.1 , we have

Proposition 6.3. If $m \geq n-1, P$ is an $m$-by-n pattern and every row of $P$ has at least 2 nonzeros, then, if $P$ contains an $(n-1)$-triangle, then $P$ is row gradual.

Proof. Since the triangle must be in a particular $(n-1)$ rows, we may reduce to the case $m=n-1$, but, then we may permute the rows and columns of $P$ so that the triangle is lower triangular and lying in the last $n-1$ columns. The row gradual property is now apparent.

We now know that row gradual is equivalent to the existence of an $(n-1)$-triangle (assuming no reduction of the $m r$ problem due to a line with only one nonzero). It 
follows that row gradual and column gradual are equivalent in the nondegenerate case. But, how does this compare to the patterns of minimal rank $n-1$ ? In an effort to prove that patterns of minimum rank $n-1$ always have an $n-1$ triangle, we encountered the following counterexample, when $n=9$.

Let the 9-by-9 pattern

$$
P=\left[\begin{array}{lllllllll}
x & x & 0 & 0 & 0 & 0 & 0 & 0 & 0 \\
x & x & 0 & 0 & 0 & 0 & x & 0 & 0 \\
0 & x & 0 & 0 & 0 & 0 & x & x & 0 \\
0 & 0 & x & x & 0 & 0 & 0 & 0 & 0 \\
0 & 0 & x & x & 0 & 0 & 0 & x & 0 \\
0 & 0 & 0 & x & 0 & 0 & 0 & x & x \\
0 & 0 & 0 & 0 & x & x & 0 & 0 & 0 \\
0 & 0 & 0 & 0 & x & x & 0 & 0 & x \\
0 & 0 & 0 & 0 & 0 & x & x & 0 & x
\end{array}\right] .
$$

Inspection of $P$ shows that it is not row (or column) gradual; thus $P$ contains no $(n-1)$-triangle (i.e 8-triangle). However, $\operatorname{mr}(P)=8$. We summarize the verification that $\operatorname{mr}(P)=8$.

Using the 1,1 entry (resp. the 4,3 and 7,5 entries) to eliminate the 1,2 and 2,1 entries (resp. the 4,4 and 5,3 entries, the 7,6 and 8,5 entries) leaves a family of patterns in which the 2,2 entry (resp. 5,4 and 8,6 entries) is (each independently) either 0 or free nonzero. The $m r$ of each these patterns is 3 plus that of a certain 6-by-6 subpattern lying in rows $2,3,5,6,8,9$ and columns $2,4,6,7,8,9$ :

$$
\left[\begin{array}{cccccc}
y_{1} & 0 & 0 & u_{1} & 0 & 0 \\
x_{1} & 0 & 0 & z_{1} & v_{1} & 0 \\
0 & y_{2} & 0 & 0 & u_{2} & 0 \\
0 & x_{2} & 0 & 0 & z_{2} & v_{2} \\
0 & 0 & y_{3} & 0 & 0 & u_{3} \\
0 & 0 & x_{3} & v_{3} & 0 & z_{3}
\end{array}\right]
$$

in which $y_{1}, y_{2}, y_{3}$ are each independently 0 or freely nonzero and all other entries freely and independently nonzero. Using $x_{i}$ to eliminate $z_{i}, i=1,2,3$, replaces $u_{i}$ by $u_{i}^{\prime}, i=1,2,3$, which are either 0 or freely nonzero, except that not both $u_{i}^{\prime}$ and $y_{i}$ may be 0 . Now using $x_{i}$ to eliminate $y_{i}$ and then $v_{i}, i=1,2,3$ leaves

$$
\left[\begin{array}{cccccc}
0 & 0 & 0 & u_{1}^{\prime} & v_{1}^{\prime} & 0 \\
x_{1} & 0 & 0 & 0 & 0 & 0 \\
0 & 0 & 0 & 0 & u_{2}^{\prime} & v_{2}^{\prime} \\
0 & x_{2} & 0 & 0 & 0 & 0 \\
0 & 0 & 0 & v_{3}^{\prime} & 0 & u_{3}^{\prime} \\
0 & 0 & x_{3} & 0 & 0 & 0
\end{array}\right]
$$


in which $u_{i}^{\prime}$ and $v_{i}^{\prime}$ cannot be simultaneously 0 , but are otherwise free. The $m r$ of this family of pattern is then 3 plus that of

$$
\left[\begin{array}{ccc}
u_{1}^{\prime} & v_{1}^{\prime} & 0 \\
0 & u_{2}^{\prime} & v_{2}^{\prime} \\
v_{3}^{\prime} & 0 & u_{3}^{\prime}
\end{array}\right]
$$

But for this, then, $m r$ is easily seen to be 2 , so that for $P$ it is 8 .

\section{References}

[1] C. R. Johnson and D. P. Stanford, Patterns that allow given row and column sums, Linear Algebra and its Applications 311 (2000), 97-105.

[2] H. Ryser, Combinatorial Mathematics, Mathematical Association of America, 1963. The Carus mathematical monographs, no. 14. 